\title{
Independence and interdependence predict health and wellbeing: divergent patterns in the United States and Japan
}

\author{
Department of Psychology, University of Michigan, Ann Arbor, MI, USA \\ 2 Department of Communication, Tokyo Woman's Christian University, Tokyo, Japan \\ ${ }^{3}$ Department of Psychology, Stanford University, Stanford, CA, USA \\ ${ }^{4}$ Department of Psychology, University of Wisconsin, Madison, WI, USA
}

Shinobu Kitayama ${ }^{1 *}$, Mayumi Karasawa ${ }^{2}$, Katherine B. Curhan ${ }^{3}$, Carol D. Ryff ${ }^{4}$ and Hazel Rose Markus ${ }^{3}$

\section{Edited by:}

Steven Heine, University of British

Columbia, Canada

\section{Reviewed by:}

Eunkook Suh, Yonsei University,

South Korea

Adam Cohen, Arizona State University, USA

\section{${ }^{*}$ Correspondence:}

Shinobu Kitayama, Department of

Psychology, University of Michigan,

Ann Arbor, MI 48103, USA.

e-mail: kitayama@umich.edu
A cross-cultural survey was used to examine two hypotheses designed to link culture to wellbeing and health. The first hypothesis states that people are motivated toward prevalent cultural mandates of either independence (personal control) in the United States or interdependence (relational harmony) in Japan. As predicted, Americans with compromised personal control and Japanese with strained relationships reported high perceived constraint. The second hypothesis holds that people achieve wellbeing and health through actualizing the respective cultural mandates in their modes of being. As predicted, the strongest predictor of wellbeing and health was personal control in the United States, but the absence of relational strain in Japan. All analyses controlled for age, gender, educational attainment, and personality traits. The overall pattern of findings underscores culturally distinct pathways (independent versus interdependent) in achieving the positive life outcomes.

\section{INTRODUCTION}

How people arrive at a sense of wellbeing including an appropriate sense of the self and good quality social ties varies with the sociocultural context (Diener et al., 1999; Kitayama and Markus, 2000; Westerhof et al., 2001). This literature indicates that the socioculturally constructed environment (composed of patterns of meanings, practices and products) provides blueprints for what it is that people are expected to do in their life and how they are expected to do so. We therefore expect that there are multiple socioculturally distinct pathways to wellbeing. Two such pathways have been characterized as independent and interdependent (Kitayama and Markus, 2000; Greenfield et al., 2003). Drawing on this overarching hypothesis, the present work investigated the cultural patterning of independence and interdependence in Japan and the US, and further probed the links of these cultural blueprints to reported wellbeing and health.

Markus, Kitayama, and colleagues have suggested that different cultural contexts value, sanction, and foster independence and interdependence to varying degrees in organizing social practices (e.g., scripted patterns of social interaction), institutions (e.g., systems of employment and promotion), and public meanings (e.g., icons and symbols) in many different domains of life (Kitayama and Uskul, in press; Markus and Kitayama, 1991, 2010). By attuning themselves to the structured set of cultural practices and meanings, individuals develop correspondingly different modes of engaging in a variety of actions - both personal (e.g., expressing the self) and interpersonal (e.g., relating to others and being friends with them). According to this analysis, the constructs of independence and interdependence refer to culturally divergent systems of action or modes of being. Specifically, the independent mode of being (which is fostered in American cultural contexts) emphasizes pursuit of personal goals and an attendant sense of the self as efficacious and in-control, while the interdependent mode of being (which is fostered in Asian cultural contexts) highlights responsiveness to the needs and expectations of social others and a resulting sense of the self as harmoniously connected to others with minimal strain and tension.

The aim of the present work is to shed a new light on the link between culture and wellbeing. Building on an extensive literature that conceptualizes motivation and goals as vital aspects of the self-system or modes of being (e.g., Greenwald, 1982; Gollwitzer and Wicklund, 1985; Cantor and Kihlstrom, 1987), we have two primary hypotheses. First, we hypothesize that goals derive from culturally prevalent ideas and practices and that people are motivated toward these primary cultural mandates, namely, personal control (and thus independence) in the United States and relational harmony (and thus interdependence) in Japan. We will test this motivation hypothesis by assessing the degree to which people failing to achieve the respective cultural mandates feel constrained and report that they "cannot do what they want to do." Second, we will also test the hypothesis that people achieve wellbeing and health in part through approaching their respective cultural mandates. Whereas in American cultural contexts wellbeing and health will be realized through high levels of personal control and mastery, in Japanese cultural contexts wellbeing and health will be realized through high levels of relational harmony.

In testing these ideas, we use multiple scales to index independence and interdependence. Our analysis emphasizes personal control as a central constituent of the independent mode of being. As a measure of independence we used two scales, one focusing on personal mastery (Lachman and Weaver, 1998) and the other on perceived control in different life domains. A newly developed scale assessing perceived control in different life domains. Further, our analysis highlights relational harmony as a crucial element of the interdependent mode 
of being. As a measure of interdependence we used scales indexing relational harmony with significant others such as family members and friends and a scale indexing relational strain with these others.

\section{CULTURAL MOTIVATIONS TOWARD INDEPENDENCE AND INTERDEPENDENCE}

The first hypothesis states that Americans are strongly motivated toward independence and Japanese are strongly motivated toward interdependence. In testing it, we draw on the well-established principle that when people are motivated toward given goal states including independence from others or interdependence with others, the motivation may be particularly evident when the goal is blocked. This principle is applicable to a variety of domains from animal behaviors to human social motivations. In a well-conducted field experiment, for example, Harris (1974) demonstrated that people in a waiting line are highly frustrated (often resulting in aggressive behaviors) when someone cuts into the line (thereby interfering with their goal attainment) and this was especially so when they were near the front (as opposed to the end) of the line. When people are near the front of the line the goal gradient is assumed to be quite steep and the drive toward the goal is high (see Berkowitz, 1989, for a review).

On the basis of this literature, one might expect that people should feel especially restrained and frustrated when the culturally sanctioned goals of either independence or interdependence are blocked. So far, we know of no data that directly speak to this possibility even with standard college student samples. We thus tested whether people would in fact experience a degree of restraint and feel that they "could not do what they wanted to do" when a key cultural motivation was blocked. We expected that Americans would feel most constrained when their pursuit of personal control was blocked. In contrast, they would not feel particularly constrained when they failed to attain relational harmony or to avoid relational strain. Given that the ideas and practices of interdependence are not as widely distributed in American contexts as in Japanese contexts, many Americans would not be as strongly motivated toward interdependence and, therefore, would not feel thwarted as much as Japanese under such conditions of blocked interdependence. Conversely, we expected that Japanese would experience a degree of constraint and feel that they "could not do what they wanted to do" when their goals of promoting relational harmony and/or avoiding relational strain were blocked. In contrast, they would not feel particularly constrained when they were prevented from attaining personal control or mastery. Japanese would not be strongly motivated toward independence and, as a consequence, they would not be frustrated as much under such conditions of blocked independence.

To measure the sense of constraint that arises when one's important cultural goals and concerns are blocked and disrupted, we used a scale designed to assess the degree to which people chronically experience constraint (called the perceived constraint scale; Pearlin and Schooler, 1978; Lachman and Weaver, 1998). Sample items include "I cannot do what I want to do" and "There is really no way I can solve the problems I have."

\section{CULTURAL PATHWAYS TO WELLBEING}

Whereas our first hypothesis was focused on motivation toward independence or interdependence, our second hypothesis was concerned with predictors of wellbeing and health. Because North
Americans are strongly motivated toward personal control and, thus, independence in general (our first hypothesis), they would also be expected to achieve wellbeing and health through the pathway of independence, that is, that of maintaining and enhancing personal control. Although important, promotion of relational harmony or avoidance of relational strain is considered to be more discretionary. In fact, such relational goals are often secondary, particularly when they are in direct conflict with the personal goals of attaining the sense of control and mastery (Heckhausen and Schultz, 1995).

Several studies have demonstrated that a strong sense of personal control is positively predictive of wellbeing and health in the United States (e.g., Lachman and Weaver, 1998; Heckhausen and Schulz, 1999). In addition, the sense of personal control is likely to yield a strong sense of self-worth or high self-esteem. A large number of studies have shown that self-esteem and, thus, an attendant tendency to self-enhance through social comparison are major predictors of wellbeing among Americans (e.g., Kwan et al., 1997; Taylor and Brown, 1988; Diener and Diener, 1995; Uchida et al., 2008). Likewise, positive feelings based on personal success such as pride and feelings of superiority are highly associated with general happiness among Americans (Kitayama et al., 2000, 2006b). Additionally, Oishi and Diener (2001) report that attaining personal goals (e.g., doing what one wants to do) leads to an enhanced wellbeing among European Americans.

In contrast, Asians are motivated toward relational harmony and, thus, interdependence in general (our first hypothesis). Further, unlike Americans, who link happiness to personal happiness, Asians associate happiness with relational harmony (Uchida and Kitayama, 2009). We therefore expected that they would achieve wellbeing and health through the interdependent pathway, that is, by promoting relational harmony and/or avoiding relational strain (Morling et al., 2002). Although important, attaining and maintaining the sense of personal control and mastery is not considered essential or primary. Indeed, especially when they are in direct conflict with relational concerns and goals, they must be subordinated under the ever-important interdependent concerns and goals. Those failing to do so are often considered as immature and childish (Lebra, 1976; Kondo, 1990).

As may be expected, wellbeing in Asian contexts is strongly predicted by social relational factors such as social harmony (Kwan et al., 1997; Kang et al., 2003), attainment of relational goals (Oishi and Diener, 2001), socially engaging emotions such as friendly feelings and feelings of respect (Kitayama et al., 2000, 2006b), and perceived emotional support from close others (Uchida et al., 2008). The hypothesized link of relational harmony with wellbeing among Asians has also been suggested by the finding that attaining relational goals (e.g., meeting expectations of significant others) is related closely to an enhanced wellbeing among Asian Americans and Japanese, but not among European Americans (Oishi and Diener, 2001).

To extend the above evidence on cross-cultural variation in wellbeing, the present work addressed three issues. First, currently available cross-cultural evidence comes nearly exclusively from samples of college students, which leaves open the applicability of the cultural psychological theories to wider populations. We thus tested age-heterogeneous samples of adults in both Japan and 
the United States. This is the first effort we know of to extend the current knowledge on cultural variation in self and wellbeing to such samples of age and socioeconomically diverse adults from different countries.

Second, one important shortcoming of existing cross-cultural work in this area is that nearly all studies focus exclusively on psychological measures of wellbeing, with scant attention paid to health outcomes. We filled this gap in the literature by incorporating reports of physical health and somatic symptoms. Furthermore, we broadened the assessment of wellbeing to include not only general evaluative judgments of wellbeing (e.g., life satisfaction; Kwan et al., 1997; Kang et al., 2003), or overall hedonic wellbeing (e.g., reported happiness, Kitayama et al., 2000, 2006b), but also eudaimonic wellbeing, which involves assessments of purpose and meaning in life, personal growth, and self-acceptance (Ryff and Keyes, 1995).

Third, although wellbeing and health have been frequently predicted by individual difference variables, such as personality traits (Schmutte and Ryff, 1997; Diener and Lucas, 1999) and educational attainment (Lachman and Weaver, 1998), contemporary work on culture and wellbeing rarely includes these variables in analytic designs, leaving open the possibility that observed cultural variations might be confounded by cultural differences in personality profiles (McCrae et al., 1998) and educational attainment. We thus controlled for both self-assessed traits and educational attainment in tests of the above relationships so as to sharpen the focus on the constructs of independence and interdependence as predictors of wellbeing and health.

\section{MATERIALS AND METHODS PARTICIPANTS AND PROCEDURE}

We recruited a convenience sample of 482 adult Japanese in midlife (199 males and 283 females; age range $=26-74$; mean age $=51$ ) both in Tokyo (the capital and the largest metropolitan area in Japan) and Sapporo (a regional center of a Northern island of Japan - Hokkaido). In Tokyo participants were recruited through adult education classes offered by the pertinent municipalities, whereas in Sapporo they were recruited through college students, who requested their adult family members (mostly parents and grandparents but in some cases older siblings) to fill out the questionnaire. All participants completed a self-administered questionnaire at home and returned it after a week. While not representative, the Japanese sample covers a wide age range and varied educational attainment levels, and importantly, was not based on college students, the most common group sampled in the current literature.

Data from this Japanese sample was compared with the national survey of Midlife Development in the US (MIDUS), which is comprised of 3032 adults (1471 males and 1561 females; age range $=25-74$; mean age $=47)$. Respondents were recruited through random-digit dialing procedures and then participated in a telephone interview, followed by the completion of self-administered questionnaires, which were returned by mail. Whereas $29.6 \%$ of Americans had a college degree or higher, $32.8 \%$ of Japanese had comparable levels of educational attainment. The percent of people who were married was far greater in Japan than in the US (92\% vs. 64\%). All scales, originally developed in English, were translated into Japanese. Back-translation was used to achieve equivalent meanings in the two languages. The Japanese version was used in Japan.

\section{MEASURES}

The questionnaires used in each country included scales designed to measure (1) independence, (2) interdependence, (3) perceived constraint, and (4) wellbeing and health. Independence was assessed with scales of personal mastery (four items, Pearlin and Schooler, 1978; Lachman and Weaver, 1998; $\alpha=0.69$ and .55 for Americans and Japanese, respectively) and domain-specific control (one item each, asking the amount of control perceived for each of seven domains such as work, finance, and family; $\alpha=0.70$ and 0.67 ). Interdependence was assessed with scales designed to measure harmonious or non-conflictual social relations including (1) relational harmony with family members (four items; $\alpha=0.82$ and 0.85 ), (2) relational strain with family members (four items; $\alpha=0.80$ and 0.79 ), (3) relational harmony with friends (four items; $\alpha=0.82$ and 0.85 ), and (4) relational strain with friends (four items; $\alpha=0.80$ and 0.79; Whalen and Lachman, 2000). The chronic experience of perceived constraint was measured with an 8 -item scale developed by Lachman and Weaver (1998) ( $\alpha=0.86$ and 0.80$)$.

To assess wellbeing and health, four separate categories of measures were employed: judgments of life satisfaction, hedonic wellbeing, eudaimonic wellbeing, and physical health. Life satisfaction was assessed with four items measuring satisfaction in different domains; $\alpha=61$ and 67); whereas hedonic wellbeing was measured with affect balance computed by subtracting an average score of 6 negative affect ratings from that of 6 positive affect ratings; Mrozek and Kolarz, 1998; negative affect $\alpha=0.87$ and 0.87 , positive affect $\alpha=0.91$ and 0.94 ). Eudaimonic wellbeing was measured by computing the average of the scale scores for four of six dimensions of psychological wellbeing (PWB, Ryff and Keyes, 1995; self-acceptance, purpose in life, personal growth, and autonomy). The remaining two PWB dimensions (environmental mastery and positive relation with others) were excluded because of conceptual overlap with the independence and interdependence predictor variables. Finally, indices of physical health included: (1) subjective health (1 item), (2) number of chronic health problems (maximum of 29; reverse-coded, e.g., diabetes), (3) frequency of somatic symptoms (maximum of 11; reverse-coded, e.g., headaches), and (4) sensitivity to unpleasant sensations (called somatic amplification, 5 items, reverse-coded; Barsky et al., 1988; $\alpha=0.55$ and 0.56). A principal component factor analysis performed on these health measures showed a robust single factor solution in both cultures. All factor loadings were substantial $(>0.60)$. The factor score was used as our measure of health.

We used gender and age as control variables as well as the five personality trait scores of agreeableness (assessed with five trait adjectives taken from John, $1990 ; \alpha=0.80$ and 0.84 ), extraversion (five adjectives; $\alpha=0.78$ and 0.82 ), neuroticism (four adjectives; $\alpha=0.74$ and 0.68 ), conscientiousness (four adjectives; $\alpha=0.58$ and 0.39 ), and openness to experience (seven adjectives; $\alpha=0.77$ and 0.82 ). All scales had reasonable reliabilities in both countries, with an exception of the Japanese $\alpha$ for conscientiousness $(=0.39)$.

\section{RESULTS}

Our analysis proceeded in three steps. First, we dimensionalized independence and interdependence. Second, we tested the first of our two major hypotheses, namely, that whereas Americans are motivated toward personal control and, more generally, toward independence, 
Japanese are motivated toward relational harmony and, more generally, toward interdependence. Third, we examined the second major hypothesis, which holds that individuals achieve wellbeing and health through living up to the major mandates of their own cultures.

\section{DIMENSIONALIZING INDEPENDENCE AND INTERDEPENDENCE}

We operationalized independence in terms of personal control (personal mastery and domain-specific control) and interdependence in terms of social harmony (the presence of positive responses from family members and friends and the absence of negative responses from family members and friends). We first performed a principal components factor analysis on the eight relevant scales within each country.

In both the US and Japan, a three-factor structure was judged to be optimal. First, three factors had eigen values of either greater than 1 or, very close to 1 . Second, in both cases, after varimax rotation, the three-factor solution was clearly interpretable and conceptually identical. We performed the same analysis on different segments of data within each country. All these analyses replicated essentially the same three-factor solution.

The American solution is summarized in the left-side panel of Table 1. The three factors were clearly interpretable as (1) "relational strain" (negative family relations, and negative friend relations), (2) "relational harmony" (positive family relations and positive friend relations), and (3) "personal control" (defined in terms of personal mastery, and domain-specific control). The control factor corresponded to our operational definition of independence, whereas the remaining two factors corresponded to our operational definition of interdependence. The Japanese solution is given in the right-side panel of Table 1 . The three factors were clearly interpretable as (1) relational harmony, (2) relational strain, and (3) personal control. For each participant, relevant scale means were averaged to yield scores for personal control, relational harmony, and relational strain.

\section{MOTIVATION TOWARD PERSONAL CONTROL AND RELATIONAL HARMONY}

If the Japanese were truly motivated toward relational harmony, they would experience a sense of constraint (e.g., "I cannot do what I want to do") when they were blocked from attaining

Table 1 | Factor analysis performed on two variables assumed to measure independence and four variables assumed to measure interdependence. In both countries, one independence factor (personal control) and two interdependence factors (relational harmony and relational strain) were identified.

\begin{tabular}{|c|c|c|c|c|c|c|}
\hline & \multicolumn{3}{|c|}{ US } & \multicolumn{3}{|c|}{ Japan } \\
\hline & 1 & 2 & 3 & 1 & 2 & 3 \\
\hline Mastery & 0.03 & 0.06 & 0.88 & 0.02 & -0.10 & 0.80 \\
\hline Control & -0.20 & 0.24 & 0.75 & 0.12 & 0.11 & 0.75 \\
\hline Family support & -0.26 & 0.79 & 0.11 & 0.84 & -0.16 & 0.04 \\
\hline Family strain & 0.81 & -0.26 & -0.05 & -0.18 & 0.85 & -0.03 \\
\hline Friend support & 0.03 & 0.82 & 0.16 & 0.79 & 0.28 & 0.13 \\
\hline Friend strain & 0.86 & 0.03 & -0.09 & 0.38 & 0.74 & 0.05 \\
\hline$\%$ Variance & 37 & 20 & 15 & 30 & 22 & 17 \\
\hline
\end{tabular}

Factor loadings that are greater than 0.7 are marked in bold. it, while Americans would feel constrained when they were prevented from attaining what they were habitually aiming at, namely, a strong sense of personal control, efficacy, mastery, and self-esteem. We used a step-wise regression to predict the perceived constraint score as a function of personal control, relational harmony, and relational strain. In the first step in the regression, four demographic variables (gender, age, culture, and educational attainment [college degree or higher vs. some college or lower) were entered. In the second step we entered five personality traits. Third, we entered the three variables pertaining to independence (personal control) and interdependence (relational harmony and relational strain). In the last step, three interaction terms between the independence/interdependence variables and culture were entered.

As can be seen in Table 2, each step accounted for a significant amount of variance. Regarding the control variables, women were more likely than men to experience constraint and perceived constraint also tended to increase as a function of age while decreasing with educational attainment. Perceived constraint also tended to be higher for Japanese than for Americans. Both agreeableness and neuroticism were positively associated with perceived constraint, while conscientiousness and openness to experience were negatively associated with perceived constraint. Higher levels of educational attainment were associated with a reduced sense of constraint. Interestingly, however, this effect was observed only among Americans, $\beta=-0.09, t_{(2890)}=6.29, p<0.0001$. Among Japanese, the effect was no different from zero, $\beta=0.03, t<1$. The interaction between culture and educational attainment was significant, $\beta=0.051, t_{(3349)}=2.55, p<0.02$.

Most importantly, and beyond the effects of these control variables, the variables related to the cultural mandates of independence and interdependence had systematic effects on perceived constraint and, moreover, these effects were often moderated by culture.

Table 2 | Summary of regression predicting perceived constraint as a function of demographics, personality, self, and culture and self.

\begin{tabular}{lcc}
\hline & Beta & $\boldsymbol{R}^{2}$ increase \\
\hline Culture & $-0.092^{* * * *}$ & $0.046^{* * * *}$ \\
Education & $-0.083^{* * * *}$ & \\
Gender & $0.032^{*}$ & \\
Age & $0.112^{* * * *}$ & $0.220^{* * * *}$ \\
Agreeableness & $0.093^{* * * *}$ & \\
Extraversion & $-0.045^{*}$ & \\
Neuroticism & $0.219^{* * * *}$ & \\
Conscientiousness & $-0.127^{* * * *}$ & $0.123^{* * * *}$ \\
Openness to experience & $-0.066^{* * * *}$ & \\
Personal control & $-0.246^{* * * *}$ & \\
Relational harmony & $-0.152^{* * * *}$ & $0.009^{* * * *}$ \\
Relational strain & $0.138^{* * * *}$ & \\
Cul $\times$ P control & $0.146^{* * * *}$ & \\
Cul $\times$ R harmony & $-0.03^{* *}$ & \\
Cul $\times$ R strain & $0.081^{* * * *}$ & \\
& &
\end{tabular}

$\times p<0.10,{ }^{*} p<0.05,{ }^{* *} p<0.01,{ }^{* *} p<0.001,{ }^{* * * *} p<0.0001$. 


\section{Motivation toward personal control}

The effect of personal control was negative in both cultures, with personal control associated with a lesser degree of perceived constraint. As predicted, the culture $\times$ personal control interaction was highly significant. See the right panel of Figure 1, which depicted the expected amount of perceived constraint for those with personal control scores that are either $1 \mathrm{SD}$ above or $1 \mathrm{SD}$ below the mean within each culture $(=0)^{1}$. The effect of personal control was highly significant in the US, $\beta=-0.37, t_{(2898)}=21.84, p<0.0001$, which is substantially stronger than the one in Japan. In fact the corresponding effect in Japan was only marginally significant, $\beta=-0.09$, $t_{(455)}=1.83, p<0.07$. This pattern lends support to the hypothesis that Americans are strongly motivated toward personal control and, thus, toward independence in general. The corresponding evidence for Japanese was weak at best.

\section{Motivation toward relational harmony and strain}

The effects of relational harmony and relational strain on perceived constraint are illustrated in the center panel and the left panel of Figure 1, respectively. As expected, perceived constraint was negatively associated with relational harmony whereas it was positively

${ }^{1}$ All three predictor variables (personal control, relational harmony, and relational strain) had lower means in Japan than in the US. As a consequence, adjusted means that control for the cultural differences in the mean levels in these variables become highly misleading in that the perceived constraint of Japanese would look unrealistically low and the perceived constraint for Americans would look unrealistically high. To avoid this misleading impression, the $x$-axis in Figure 1 is centered around the mean of the pertinent predictor variable within each culture. In this way, the figure accurately represents both the culturally variable levels of perceived constraint and the slope within each culture as a function of each of the three predictor variables. This caveat also applies to Figure 2. associated with relational strain. In both the US and Japan, relational harmony was reliably associated with (the absence) of perceived constraint, $\beta=-0.10, t_{(2890)}=6.20, p<0.001$ and $\beta=-0.22, t_{(452)}=4.80$, $p<0.001$, respectively, but the effect of relational harmony was somewhat stronger for Japanese than for Americans although the difference was statistically trivial (see Table 2). In contrast, the interaction with culture was highly significant for relational strain. The effect of relational strain was positive both in the US and in Japan, $\beta=0.06, t_{(2890)}=3.55, p<0.001$ and $\beta=0.24, t_{(452)}=5.54, p<0.0001$, respectively. As predicted, it was significantly stronger in Japan than in the US. This pattern supports the hypothesis that Japanese are, in fact, more strongly motivated than Americans toward relational harmony and, thus, toward independence in general.

It is curious that the strong relational motivation for Japanese was especially evident for the measure of relational strain, suggesting that it is the failure of avoiding relational strain that creates the sense of constraint among Japanese. In contrast, the effect of relational harmony was comparatively weak. This converges with prior work that demonstrates a greater prevention (rather than promotion) orientation for Asians than for European Americans (Elliot et al., 2006). The finding is also consistent with the notion that Japanese seek to self-improve by setting their standards high and avoiding problems and shortcomings relative to such standards (Heine et al., 2001). The corresponding evidence for Americans was much weaker.

\section{PREDICTORS OF WELLBEING AND HEALTH}

The second major hypothesis of the current work is related to the first. Because Americans are strongly motivated toward independence, attaining independence is likely to be highly self-affirming for them,

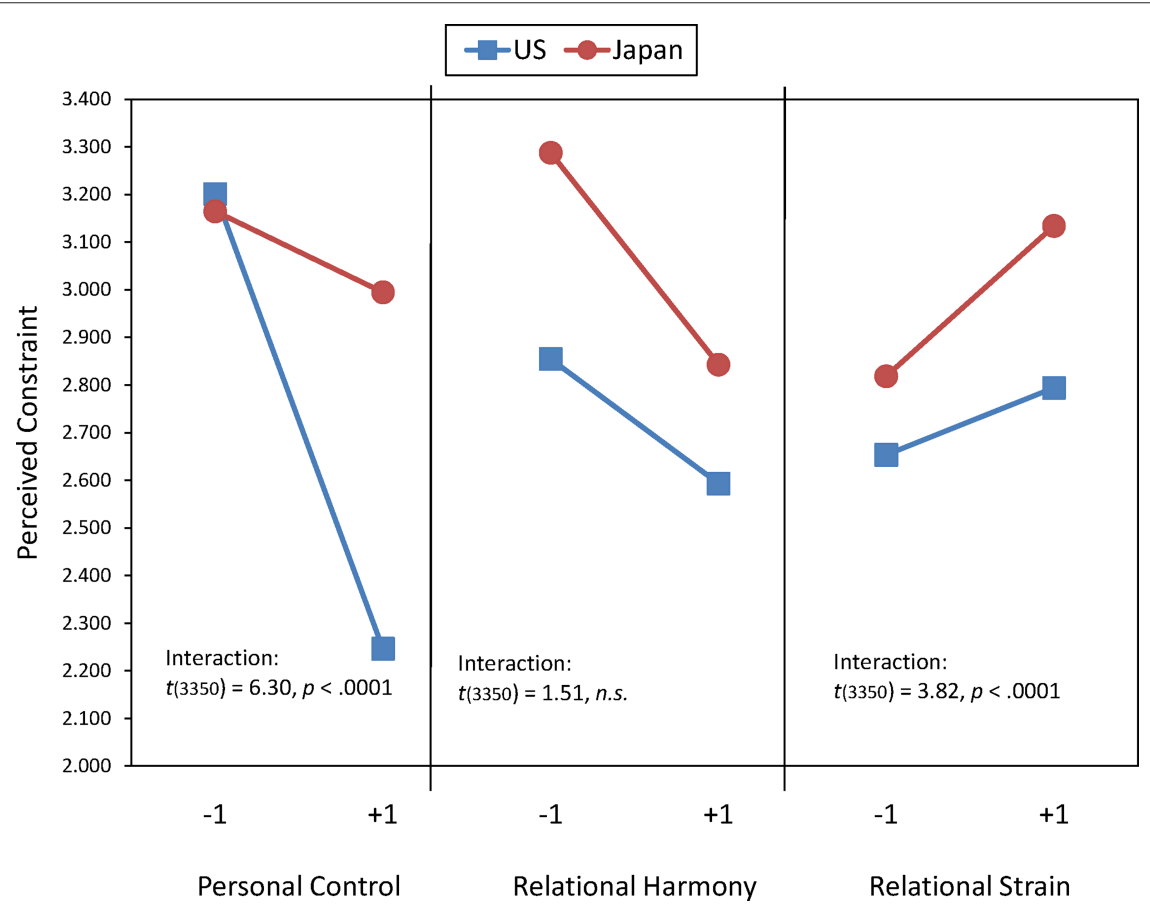

FIGURE 1 | Effects of personal control, relational harmony, and relational strain on perceived constraint in Japan and the United States. Estimated means for perceived constraints are plotted at 1 SD lower or higher than the mean level of each of the three independent variables within each culture. 
thereby enhancing wellbeing and health. Conversely, because Japanese are strongly motivated toward interdependence, attaining interdependence is likely to be highly self-affirming for them, thereby enhancing wellbeing and health. We tested these predictions after having controlled for both demographic variables and personality traits.

In a step-wise regression performed on each of the four outcome measures, demographic variables were entered first, followed by personality traits. The variables pertaining to cultural mandates were then entered. Interactions involving cultural mandate and culture were entered in the last step.

Results from the regression analysis are summarized in Table 3. In all the four cases, each step resulted in a significant increment of variance accounted. Regarding control variables, wellbeing increased as a function of age for the two generic, hedonic measures of wellbeing (life satisfaction and affect balance), while it decreased as a function of age for eudaimonic wellbeing and health. Educational attainment had positive effects on the outcome variables. The effects were significant in all cases except in affect balance. Unlike in the analysis of perceived constraint, the effects of educational attainment on health and wellbeing outcome variables did not differ between the two cultures. Further, personality traits also had systematic effects on wellbeing and health. Agreeableness had a weak, negative association with wellbeing and health. The effect of extraversion was strongly positive, as was the effect of conscientiousness. The effects of neuroticism and openness to experience were negative. The variance accounted for by personality traits was especially large for affect balance and eudaimonic wellbeing.

\section{Cultural mandates}

Above and beyond the effects of the control variables, cultural mandates had some systematic effects on wellbeing and health. As hypothesized, as personal control increased, relational harmony increased, and relational strain decreased, wellbeing and health went up. Importantly, however, these effects were moderated by location especially for personal control and relational strain. As can be seen in Table 3, both the interaction between culture and relational strain and the interaction between culture and personal control were all in the predicted directions and, often, statistically significant.

Because the pattern of the data was highly similar across the four measures of wellbeing and health, we created a single summary index of wellbeing and health. We performed a principal component analysis on the four measures of wellbeing and health, yielding a single factor solution. In both cultures, a one-factor solution was optimal. The corresponding factor score was computed on the basis of another principal component analysis performed on the entire sample and then submitted to another step-wise regression with the same set of predictor variables.

The effect of personal control is illustrated in the left panel of Figure 2. There was a strong positive association between personal control and the wellbeing and health composite in both the US and Japan, $\beta=0.40, t_{(2859)}=27.82, p<0.0001$ and $\beta=0.24, t_{(458)}=5.37$, $p<0.001$, respectively. Importantly, however, the culture $\times$ personal control interaction was significant, $\beta=-0.048, t_{(3314)}=2.42, p<0.02$. As predicted, the positive association between personal control and the wellbeing and health composite was significantly stronger for Americans than for Japanese.

The effect of relational harmony is illustrated in the center panel of Figure 2. Overall, there was a positive association between relational harmony and the wellbeing and health composite both in the US and Japan, $\beta=0.10, t_{(2859)}=7.00, p<0.01$ and $\beta=0.17$, $t_{(458)}=4.45, p<0.01$, respectively. But the interaction with culture was also significant, $\beta=0.037, t_{(3314)}=2.15, p<0.05$, indicating that the effect of harmony, as predicted, was stronger in Japan than in the US.

Table 3 | Summary of regression predicting life satisfaction, affect balance, eudaimonic wellbeing, and health as a function of demographics, personality, self, and culture and self.

\begin{tabular}{|c|c|c|c|c|c|c|c|c|}
\hline & \multicolumn{2}{|c|}{ Life satisfaction } & \multicolumn{2}{|c|}{ Affect balance } & \multicolumn{2}{|c|}{ Eudaimonic wellbeing } & \multicolumn{2}{|c|}{ Health } \\
\hline & Beta & $R^{2}$ increase & Beta & $R^{2}$ increase & Beta & $R^{2}$ increase & Beta & $R^{2}$ increase \\
\hline Culture & $0.126^{* * * *}$ & $0.193^{* * * *}$ & $0.128 * * * *$ & $0.027 * * * *$ & $0.104^{* * * *}$ & $0.299 * * * *$ & $0.178 * * * *$ & $0.055^{* * * *}$ \\
\hline Education & $0.031 * * *$ & & 0.017 & & $0.098 * * * *$ & & $0.092 * * * *$ & \\
\hline Gender & -0.008 & & $-0.041^{* *}$ & & $-0.04^{* * *}$ & & $-0.104^{* * * *}$ & \\
\hline Age & $0.094^{* * * *}$ & & $0.042 * *$ & & $-0.064^{* * * *}$ & & $-0.119 * * * *$ & \\
\hline Agreeableness & 0.018 & $0.280 * * * *$ & $-0.034 x$ & $0.355^{* * * *}$ & $-0.045^{*}$ & $0.316^{* * * *}$ & $-0.067^{* *}$ & $0.179 * * * *$ \\
\hline Extraversion & $0.143^{* * * *}$ & & $0.198 * * * *$ & & $0.078 * * *$ & & $0.108 * * * *$ & \\
\hline Neuroticism & $-0.094^{* * * *}$ & & $-0.414^{* * * *}$ & & $-0.191 * * * *$ & & $-0.281^{* * * *}$ & \\
\hline Conscientiousness & $0.09 * * * *$ & & $0.049 * *$ & & $0.212^{* * * *}$ & & $0.076 * * *$ & \\
\hline Openness to & $-0.117^{* * * *}$ & & $-0.062 * *$ & & $0.176^{* * * *}$ & & $-0.062 * *$ & \\
\hline \multicolumn{9}{|l|}{ experience } \\
\hline Personal control & $0.414^{* * * *}$ & $0.174^{* * * *}$ & $0.255^{* * * *}$ & $0.069 * * * *$ & $0.26 * * * *$ & $0.060^{* * * *}$ & $0.21 * * * *$ & $0.057 * * * *$ \\
\hline Relational harmony & $0.189 * * * *$ & & $0.113^{* * * *}$ & & $0.131^{* * * *}$ & & 0.034 & \\
\hline Relational strain & $-0.222 * * * *$ & & $-0.096^{* * *}$ & & $-0.105^{* * *}$ & & $-0.139 * * * *$ & \\
\hline Cul $\times$ P control & $-0.055^{*}$ & $0.006 * * *$ & -0.028 & $0.001 *$ & -0.022 & $0.001 * *$ & $-0.043 x$ & $0.001 \times$ \\
\hline Cul $\times$ R harmony & $0.072 * * * *$ & & 0.03 & & 0 & & 0.023 & \\
\hline Cul $\times$ R strain & $-0.115^{* * * *}$ & & $-0.055^{* *}$ & & $-0.075^{* * *}$ & & $-0.038 x$ & \\
\hline
\end{tabular}

$\times p<0.10,{ }^{*} p<0.05,{ }^{*} p<0.01,{ }^{* *} p<0.001,{ }^{* * *} p<0.0001$. 


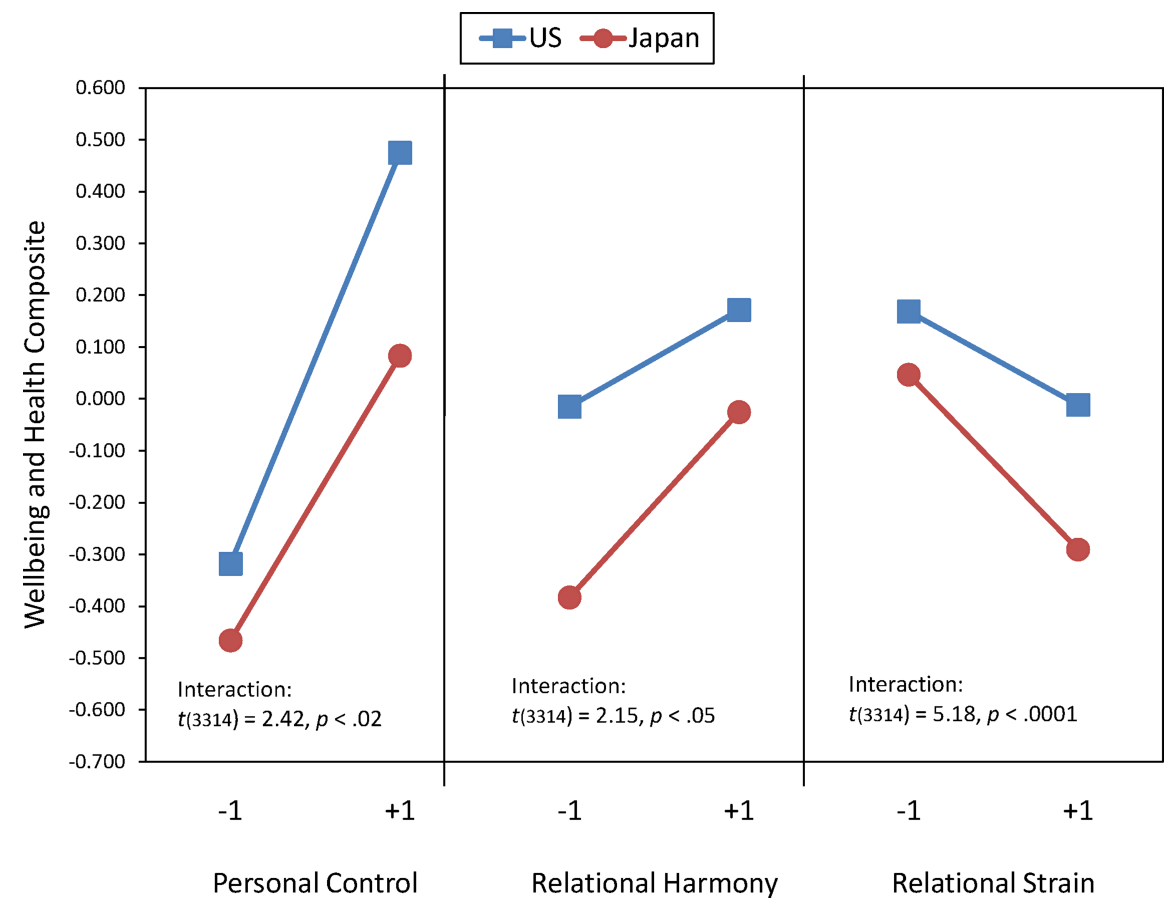

FIGURE 2 | Effects of personal control, relational harmony, and relational strain on the composite index of wellbeing and health in Japan and the United States. Estimated means for the wellbeing composite are plotted at 1 SD lower or higher than the mean level of each of the three independent variables within each culture.

The effect of relational strain is illustrated in the right panel of Figure 2. Overall, there was a strongly negative association between relational strain and the wellbeing and health composite both in the US and Japan, $\beta=-0.09, t_{(2859)}=6.57, p<0.01$ and $\beta=-0.26$, $t_{(458)}=7.07, p<0.001$. Relational strain did compromise wellbeing and health. Yet, as predicted, the interaction between culture and relational strain was highly significant, $\beta=-0.094, t_{(3314)}=5.18$, $p<0.0001$. Relative to the US data, the Japanese data showed a stronger effect of relational strain ${ }^{2}$.

\section{DISCUSSION \\ CULTURAL VARIATION IN PATHWAYS TO WELLBEING AND HEALTH}

Previous research, conducted mostly in North America, has shown that both personal control and mastery strongly predict wellbeing and health (e.g., Lachman and Weaver, 1998; Schneiderman et al., 2001). Using age-diverse community samples from both the United States and Japan, the present work demonstrated that this conclusion is contingent on culture. Obviously, biology is important in health. However, our work has begun to show that culture-specific psychological variables such as personal control and relational harmony or strain have substantial impacts on various health outcomes.

We started with the hypothesis that whereas the independent mode of being is constituted by an active motivational orientation toward control and mastery, the interdependent mode of being involves a strong motivational orientation toward

${ }^{2}$ It is important to note that the same conclusions were obtained when perceived constraint was entered and thus statistically controlled for in the regressions. This means that the effects reported here were found above and beyond those attributable to the experience of constraint that arises when people fail to live up to the cultural mandates. relational harmony (Kitayama and Markus, 2000; Markus and Kitayama, 2010). We reasoned that such motivational orientations would manifest themselves most when they were blocked. As predicted, Americans reported that they "could not do what they wanted," thus, showing a sense of constraint in their lives especially when they were low in personal control. In contrast, Japanese reported that they "could not do what they wanted," thus showing feelings of constraint especially when they were high in relational strain.

We then demonstrated culturally divergent effects of both control and relational harmony/strain on wellbeing and health. Specifically, control proved to be reliably more significant predictor of the wellbeing and health composite among Americans than among Japanese. This finding is consistent with several recent findings that demonstrate the significance of self-esteem in predicting wellbeing in individualistic cultures such as the US (Kwan et al., 1997; Diener et al., 1999; Uchida et al., 2008). Together, these findings lend strong support to the hypothesis that control and associated sense of the self as efficacious and esteemed is a central element of independence that is particularly privileged and emphasized in North America. In contrast, in Japan it was the absence of relational strain that most powerfully predicted the summary index of wellbeing and health. This finding converges with the hypothesis that in Japanese cultural contexts responsiveness to others and attendant social harmony are strongly valued and sanctioned.

In demonstrating cultural differences in the effects of both control and relational harmony/strain on perceived constraint, wellbeing, and health we controlled for major personality variables (in addition to age and gender), as well as educational attainment, in both American and Japanese samples. We believe that this is an 
important analytic step as it shows that the cultural differences we found cannot be reduced to cultural variations in these individual difference variables.

Extrapolating from the current findings, one might propose that any mismatch between prevailing cultural expectations or norms and one's psychological propensities might compromise health and wellbeing. This general hypothesis may help push the field forward beyond the constructs of independence and interdependence. For example, in a cultural group where honor is a significant virtue, one may be especially unhappy and unsatisfied if his or her honor is compromised. Further, such a person may be at risk for impaired health, physical or mental. The same idea potentially applies to other cultural norms, such as achievement ethics, egalitarianism, religious purity, and political liberalism or conservatism. These possibilities constitute rich future directions.

\section{ARE RELATIONAL FACTORS REALLY UNIMPORTANT IN INDEPENDENT CULTURE?}

The present data revealed that for Americans the effects of relational harmony or relational strain on wellbeing and health are relatively small although they were still statistically significant. This observation might seem at odds with the emerging literature, theoretical and empirical, suggesting the fundamental significance of social relations in health and wellbeing. For example, multiple large-scale epidemiological studies have demonstrated a sizable longitudinal benefit of availability of social relations on a variety of health and wellbeing outcome variables (House et al., 1988; Kawachi and Berkman, 2001).

We suspect that our findings showing relatively weak effects of relational harmony or relational strain on wellbeing and health among Americans are, in part, due to the fact that we looked at these effects after controlling for personal control. As proposed by Uchida et al. (2008), in independent cultural contexts, supportive others may be crucially important to help maintain the ever-important sense of personal control and efficacy, which, in turn, likely enhances health and wellbeing. This implies that the effects of relational harmony and strain on wellbeing are likely mediated by personal control. Indeed, when personal control was regressed on relational harmony and relational strain, the pertinent regression coefficients for relational harmony were highly significant for both Americans and Japanese, $\beta=0.33$, $t_{(2950)}=17.11, p<0.0001$ and $\beta=0.19, t_{(471)}=4.18, p<0.0005$, for Americans and Japanese, respectively. As expected, however, the American coefficient was significantly greater in magnitude than its Japanese counterpart, $\beta=0.060, t_{(3354)}=2.99, p<0.005$. In the US, relational harmony had a highly reliable effect on the wellbeing and health composite, $\beta=0.17, t_{(2860)}=11.04, p<0.0001$, but once personal control was entered, the effect of relational harmony was significantly reduced to $\beta=0.10, t_{(2859)}=7.00, p<0.0001$. The mediation by personal control was highly significant, Sobel test $=9.59, p<0.0001$. In Japan, the effect of relational harmony on the wellbeing and health composite was reduced from $\beta=0.20$, $t_{(448)}=4.96, p<0.0001$ to $\beta=0.17, t_{(447)}=4.45, p<0.0001$. The same mediation appears much weaker although still statistically significant, Sobel test $=2.26, p<0.05$. Relational strain showed no such cross-cultural difference, showing a marginally negative effect on personal control when the countries were combined, $\beta=-0.040, t_{(3421)}=1.80, p<0.08$.
This subsidiary evidence suggests that it is misleading to conclude from our results that relational factors are unimportant among Americans. To the contrary, they can be very important in sustaining their sense of control, autonomy, and independence. Thus, the relational factors are likely to have significant influences on health and wellbeing, but this influence is likely to be mediated by affirmation of the independence of the self. Here, there is a very different dynamic among Japanese. Our results show that the relational factors (enhanced harmony and reduced strain) can directly affirm the sense of interdependence and, thus, improve health and wellbeing directly without any mediation by independence-related factors such as control and self-esteem (Uchida et al., 2008).

\section{FACETS OF INDEPENDENCE AND INTERDEPENDENCE}

Our decision to focus on personal control and relational harmony to index independence and interdependence respectively was pragmatic. The American data had been collected independent of and prior to the current work. Necessarily we needed to do our best within the confines of scales included in the original American study. Quite fortunately, however, personal control and relational harmony are likely to be two central elements of the respective modes of being and, moreover, past work suggested that these are two aspects that are likely related directly to some of mental and physical health outcome variables (Diener et al., 1999; Kitayama and Markus, 2000, for reviews).

Further, in their meta-analyses of scales measuring independence and interdependence, Oyserman et al. (2002) pointed out that the existing scales of independence cover (1) valuing personal independence, (2) personal achievement, (3) self-knowledge, (4) uniqueness, (5) privacy, (6) clear communication, and (7) competition to capture independence. Personal control, as assessed herein, requires an attitude toward independence, a strong propensity toward achievement and competition, and clear selfknowledge. It seems therefore to tap some of the facets covered in the literature, although aspects such as privacy and clear communication were not addressed. Likewise, Oyserman and colleagues observed that the existing scales of interdependence cover several faces including (1) sense of duty to group, (2) relatedness to others, (3) seeking others' advice, (4) harmony, (5) working in groups, (6) sense of belonging to a group, (7) contextual self, and (8) valuing hierarchy. Many of these facets are closely linked to both relational harmony and relational strain, as assessed in the present investigation.

Future work should examine whether the index of cultural orientation suggested here would converge with other indicators of independence and interdependence (Hofstede, 1980; Schwartz, 1992; Singelis, 1994; Triandis, 1995).

\section{UNIVERSALITY AND CULTURE-SPECIFICITY OF PERSONAL CONTROL}

The current data presents an interesting test case for discerning cultural universals (Norenzayan and Heine, 2005). From our factor analysis (Table 1), it is clear that the control factor as constituted by personal mastery and domain-specific control is common in the two cultures. To the extent that control is experienced in terms of these two relevant elements and these elements only, then control may be reasonably said to be cross-culturally common and, 
perhaps, even universal. So the case can be made for a position that control is a universal building block of self-regulation (e.g., Heckhausen and Schulz, 1995; Bandura, 1996).

It is important to note, however, that Lachman and Weaver (1998) construed personal control, in part, as freedom from perceived constraints imposed by external barriers against one's goals. In support of this view, our data suggest that Americans do experience personal control as an amalgam of personal mastery, domain-specific control, and freedom from constraint. But defined this way, personal control might turn out to be relatively unique to the American culture and if this were the case, it might not travel well to Japan. Accordingly, one could argue that control is fully imbued with cultural meanings (Weisz et al., 1984; Gould, 1999; Morling and Evered, 2006).

A general lesson to be learned from this discussion may be that universality and culture-specificity are in fact two facets of the same process. Because culturally specific aspects of any given process can only be defined vis-à-vis its universal aspects, it is indispensable to take the latter into account. At the same time, the culture-specific aspects can transform the process itself in non-trivial ways. For example, the sense of control combined with the perceived freedom from constraint in the US might come with a greater sense of efficacy and agency and a more vigorous desire to take charge and to influence the surrounding. The process transformed, then, may well result in very different psychological and interpersonal consequences. Accordingly, theories that fail to give due considerations to these culture-specific aspects are incomplete at best and can even be misleading.

\section{REGIONAL VARIATION IN JAPAN: HOKKAIDO AS JAPAN'S "WILD NORTH"}

Kitayama and colleagues have amassed some empirical evidence for the hypothesis that the northern island of Hokkaido carries a "spirit of independence" because of its recent history of voluntary settlement (Kitayama et al., 2006a). To further test this notion, we compared our Hokkaido sample with its Tokyo counterpart. We observed that the effect of relational strain on the outcome variables tended to be smaller in Hokkaido than in Tokyo, as expected. Indeed, in three of the four outcome variables (life satisfaction, affect balance, and health) the regional difference was statistically significant. Further, the effect of personal control on health and wellbeing outcome variables tended to be larger in Hokkaido than in Tokyo although the difference never reached statistical significance. There was no regional difference in the effect of relational harmony. The present findings go beyond the earlier study and show that the uniqueness of Hokkaido as a "frontier" region of Japan is evident even with age-heterogeneous non-student adult samples.

The hypothesis that a history of frontier settlement encourages independence would also suggest a degree of regional variation in North America such that the independent pattern is more pronounced among families that had settled in Western states and

\section{REFERENCES}

Bandura, A. (1996). Social Foundations of Thought and Action. Englewood Cliffs, NJ: Prentice-Hall.

Barsky, A. J., Goodson, J. D., Lane, R. S., and Cleary P.D. (1988). The amplification of somatic symptoms. Psychosom. Med. 50, 510-519.
Berkowitz, L. (1989). The frustrationaggression hypothesis: an examination and reformulation. Psychol. Bull. 106, 59-73.

Cantor, N., and Kihlstrom, J. F. (1987). Personality and Social Intelligence. Englewood Cliffs, NJ: PrenticeHall.

lived there over generations, as compared to those staying in, say, New England for an equally long period of time. Some encouraging evidence is already available (Plaut et al., 2002; see Kitayama et al., 2010, for a review), but this issue deserves a more careful examination in future work.

\section{FUTURE DIRECTIONS}

Future research should examine other related facets of both independence and interdependence and refine our measurement of these constructs. For example, pursuit of personal goals and adjustment to others' needs and expectations might define another significant facet of independence and interdependence, respectively. Similar refinement and expansion are needed in the measurement of health outcomes. In addition to a variety of selfreported measures of health and somatic symptoms, it is important to include behavioral assessments of health such as smoking, drinking, and exercise.

Another significant expansion is to link the subjective self-report measures to physiological measures. In fact, an increasing number of studies have demonstrated, mostly within North American populations, that there is a physiological cost of social isolation and social rejection (Uchino et al., 1996), while others have shown that positive social relationships are linked with reduced cardiovascular risk and better neuroendocrine regulation (Ryff et al., 2004). Such investigations are valuable for bringing objectively-measured outcomes to the inquiry, thereby reducing the extent to which findings are inflated by general tendencies (at the individual level) toward positive or negative self-ratings regardless of the content being assessed. Beyond that these biological variables may provide promising new outcomes for investigating the health impact of the cultural mandates (independence and interdependence) described herein. That is, we might expect even stronger associations between social engagement or interdependence and physiological measures of wellbeing in Asia, where social relations carry a high cultural premium. Although a recent study failed to find evidence for this prediction (Seeman et al., 2004), the issue deserves further investigations with culturally valid measures of social engagement and interdependence.

All in all, then, a further empirical inquiry into the cultural patterning of independence, interdependence, and wellbeing with a variety of measures, both subjective, behavioral, and physiological has a great promise of uncovering the sociocultural underpinning of human adaptation and health.

\section{ACKNOWLEDGMENT}

This research was supported by a grant from the National Institute on Aging (5R37AG027343) to conduct a study of Midlife in Japan (MIDJA) for comparative analysis with MIDUS (Midlife in the US, P01-AG020166). We thank Jiyoung Park for her assistance in data analysis.

Diener, E., and Diener, M. (1995). Crosscultural correlates of life satisfaction and self-esteem. J. Pers. Soc. Psychol. 68, 653-663.

Diener, E., and Lucas, R. E. (1999) "Personality and subjective wellbeing," in Wellbeing: The Foundations of Hedonic Psychology, eds D. Kahneman,
E. Diener, and N. Schwarz (New York: Russell Sage Foundation), 213-229.

Diener, E., Suh, E. M., Lucas, R. E., and Smith, H.L. (1999). Subjective wellbeing: three decades of progress. Psychol. Bull. 125, 276-302.

Elliot, A. J., Chirkov, V. T., Kim, Y., and Sheldon, K.M. (2006).A cross-cultural 
analysis of avoidance (relative to approach) personal goals. Psychol. Sci. 12, 505-510.

Gollwitzer, P. M., and Wicklund, R. A. (1985). "The pursuit of self-defining goals," in Action Control: From Cognition to Behavior, eds J. Kuhl and J. Beckmann (Heidelberg: SpringerVerlag), 61-85.

Gould, S. J. (1999). A critique of Heckhausen and Schulz's (1995) lifespan theory of control from a crosscultural perspective. Psychol. Rev. 106, 597-604.

Greenfield, P. M., Keller, H., and Fuligini, A. (2003). Cultural pathways through universal development. Annu. Rev. Psychol. 54, 461-490.

Greenwald, A. G. (1982). "Ego task analysis: a synthesis of research on egoinvolvement and self-awareness," in Cognitive Social Psychology, eds A. H. Hastorf and A. M. Isen (New York: Elsevier/North-Holland), 109-147.

Harris, M. B. (1974). Mediators between frustration and aggression in a field experiment. J. Exp. Soc. Psychol. 10, 561-571.

Heckhausen, J., and Schulz, R. (1995). A life-span theory of control. Psychol. Rev. 102, 284-304.

Heine, S. J., Kitayama, S., Lehman, D. R., Takata, T., Ide, E., Lueng, C., and Matsumoto, H. (2001). Divergent consequences of success and failure in Japan and North America: an investigation of self-improving motivations and malleable selves. J. Pers. Soc. Psychol. 81, 599-615.

Hofstede, G. (1980). Culture's Consequences: International Differences in Work-Related Values. Beverly Hills, CA: Sage.

House, J. S., Landis, K. R., and Umberson, D. (1988). Social relationships and health. Science $241,540-545$.

John, O. P. (1990). 'The "Big Five" factor taxonomy: dimensions of personality in the natural language and in questionnaires', in Handbook of Personality: Theory and Research, ed. Pervin (New York: Guilford Press), 66-100.

Kang, S., Shaver, P. R., Min, K., and Jin, H. (2003). Culture-specific patterns in the prediction of life satisfaction: roles of emotion, relationship quality, and self-esteem. Pers. Soc. Psychol. Bull. 29, 1596-1608.

Kawachi, I., and Berkman, L. (2001). Social ties and mental health. J. Urban Health 78, 458-467.

Kitayama,S., Conway,L. G., Pietromonaco, P. R., Park, H., and Plaut, V. C. (2010). Ethos of independence across regions in the United States: the productionadoption model of cultural change. Am. Psychol. 65, 559-574.
Kitayama, S., Ishii, K., Imada, T., Takemura, K., and Ramaswamy, J. (2006a). Voluntary settlement and the spirit of independence: evidence from Japan's "Northern Frontier". J. Pers. Soc. Psychol. 91, 369-384.

Kitayama, S., Mesquita, B., and Karasawa, M. (2006b). Cultural affordances and emotional experience: socially engaging and disengaging emotions in Japan and the United States. J. Pers. Soc. Psychol. 91,890-903.

Kitayama, S., and Markus, H. R. (2000). "The pursuit of happiness and the realization of sympathy: Cultural patterns of self, social relations, and wellbeing," in Culture and Subjective Wellbeing, Eds E. Diener and E. M. Suh (Cambridge, MA: MIT Press), 113-1621.

Kitayama, S., Markus, H.R., and Kurokawa, M. (2000). Culture, emotion and wellbeing: good feelings in Japan and the U.S. Cogn. Emot. 14, 93-124.

Kitayama, S., and Uskul, A. (in press). (Culture), mind, and the brain: current evidence and future directions. Annu. Rev. Psychol.

Kondo, D. K. (1990). Crafting Selves: Power, Gender, and Discourses of Identity in Japanese Workplace. Chicago: University of Chicago Press.

Kwan, V. S. M., Bond, M. H., and Singelis, T. M. (1997). Pancultural explanations for life satisfaction: adding relationship harmony to self-esteem. J. Pers. Soc. Psychol. 73, 1038-1051.

Lachman, M. E., and Weaver, S. L. (1998). The sense of control as a moderator of social class differences in health and wellbeing. J. Pers. Soc. Psychol. 74, 763-773.

Lebra, T. S. (1976). Japanese Patterns of Behavior. Honolulu: University of Hawaii Press.

Markus, H. R., and Kitayama, S. (2010). Culture and self: a cycle of mutual constitution. Perspect. Psychol. Sci. 5, 420-430.

Markus, H., and Kitayama, S. (1991). Culture and the self: implications for cognition, emotion, and motivation. Psychol. Rev. 98, 224-253.

McCrae, R. R., Yik, M. S. M., Trapnell, P. D., Bond, M. H., and Paulhus, D. L. (1998). Interpreting personality profiles across cultures: bilingual, acculturation, and peer rating studies of Chinese undergraduates. J. Pers. Soc. Psychol. 74, 1041-1055.

Morling, B., and Evered, S. (2006). Secondary control reviewed and defined. Psychol. Bull. 132, 269-296.

Morling, B., Kitayama, S., and Miyamoto, Y. (2002). Cultural practices empha- size influence in the US and adjustment in Japan. Pers. Soc. Psychol. Bull. 28, 311-323.

Mrozek, D. K., and Kolarz, C. M. (1998) The effect of age on positive and negative affect: a developmental perspective on happiness. J. Pers. Soc. Psychol. 75, 1333-1349.

Norenzayan, A., and Heine, S. J. (2005) Psychological universals: what are they and how can we know? Psychol. Bull. 135, 763-784.

Oishi, S., and Diener, E. (2001). Goals, culture, and subjective wellbeing. Pers. Soc. Psychol. Bull. 27, 1674-1682.

Oyserman, D., Coon, H. M., and Kemmelmeier, M. (2002). Rethinking individualism and collectivism: evaluation of theoretical assumptions and meta-analyses. Psychol. Bull. 128 , 3-72.

Pearlin, L. I., and Schooler, C. (1978) The structure of coping. J. Health Soc Behav. 19, 2-21.

Plaut, V. C., Markus, H. R., and Lachman, M. E. (2002). Place matters: consensual features and regional variation in American well-being and self. J. Pers. Soc. Psychol. 83, 160-184.

Ryff, C. D., and Keyes, C. L. M. (1995) The structure of psychological wellbeing revisited. J. Pers. Soc. Psychol. 69 719-727.

Ryff, C. D., Singer, B. H., and Love, G. D. (2004). Positive health: connecting wellbeing with biology. Philos. Trans. R. Soc. Lond. B, 259, 1383-1394.

Schwartz, S. H. (1992). "Universals in the content and structure of values: theoretical advances and empirical tests in 20 countries," in Advances in Experimental Social Psychology, ed. M Zanna (San Diego: Academic Press), $1-65$.

Schmutte, P. S., and Ryff, C. D. (1997) Personality and wellbeing: re-examining methods and meanings. J. Pers. Soc. Psychol. 73, 549-559.

Schneiderman, N., Antoni, M. H., Saab, P. G., and Ironson, G. (2001). Health psychology: psychosocial and biobehavioral aspects of chronic disease management. Annu. Rev. Psychol. 52, 555-580.

Seeman, T., Glei, D., Goldman, N. Weinstein, M., Singer, B., and Yu-Hsuan, L. (2004). Social relationships and allostatic load in Taiwanese elderly and near elderly. Soc. Sci. Med. 59, 2245-2257.

Singelis, T. M. (1994). The measurement of independent and interdependent self-construals. Pers. Soc. Psychol. Bull. 20, 580-591.

Taylor, S. E., and Brown, J. D. (1988). Illusion and wellbeing: a social psychological perspective on mental health. Psychol. Bull. 103, 193-210.

Triandis, H. C. (1995). Individualism and Collectivism. Boulder: Westview Press

Uchida, Y., and Kitayama, S. (2009). Happiness and unhappiness in east and west. Emotion 9, 441-456.

Uchida, Y., Kitayama, S., Mesquita, B., Reyes, J., and Morling, B. (2008). Is perceived emotional support beneficial?: Wellbeing and health in independent and interdependent cultures. Pers. Soc. Psychol. Bull. 34, 741-754.

Uchino, B. N., Cacioppo, J. T., and Kiecolt-Glaser, J. K. (1996). The relationship between social support and physiological processes: a review with emphasis on underlying mechanisms and implications for health. Psychol. Bull. 119, 488-531.

Whalen, H. R., and Lachman, M. E. (2000). Social support and strain from partner, family, and friends: costs and benefits for men and women. J. Soc. Pers. Relat. 17, 5-30.

Weisz, J. R., Rothbaum, F. M., and Blackburn, T. C. (1984). Standing out and standing in: the psychology of control in America and Japan. Am. Psychol. 39, 955.

Westerhof, G. J., Katzko, M.W., DittmannKohli, F., and Hayslip, B. (2001). Life contexts and health-related selves in old age: perspectives from the United States, India, and Congo/Zaire. J. Aging Stud. 15, 105-126.

Conflict of Interest Statement: The authors declare that the research was conducted in the absence of any commercial or financial relationships that could be construed as a potential conflict of interest.

Received: 10 July 2010; paper pending published: 11 August 2010; accepted: 14 September 2010; published online: 02 December 2010.

Citation: Kitayama S, Karasawa $M$, Curhan KB, Ryff CD and Markus HR (2010) Independence and interdependence predict health and wellbeing: divergent patterns in the United States and Japan. Front. Psychology 1:163. doi: 10.3389/ fpsyg.2010.00163

This article was submitted to Frontiers in Cultural Psychology, a specialty of Frontiers in Psychology.

Copyright (c) 2010 Kitayama, Karasawa, Curhan, Ryff and Markus. This is an open-access article subject to an exclusive license agreement between the authors and the Frontiers Research Foundation, which permits unrestricted use, distribution, and reproduction in any medium, provided the original authors and source are credited. 